Bundesgesundheitsbl 2020 - 63:570-576 https://doi.org/10.1007/s00103-020-03132-9 Online publiziert: 7. April 2020

(c) Der/die Autor(en) 2020

\author{
Carl-Hermann Hempen ${ }^{1}$ Josef Hummelsberger ${ }^{2}$ \\ ${ }^{1}$ Technische Universität München, München, Deutschland \\ ${ }^{2}$ München, Deutschland
}

\title{
Traditionelle Chinesische Medizin (TCM) - vom Mythos zur Evidenz
}

4. Bewegungstherapien (Taijiquan und Qigong) und

5. Tuina (chinesische manuelle Medizin).

In China entstand vor ca. 2500 Jahren ein eigenes Medizinsystem. Basierend auf den kulturellen, philosophischen und erkenntnistheoretischen Prinzipien der damaligen chinesischen Philosophie entwickelten sich eigene Vorstellungen von Gesundheit und Kranksein. Dies bis heute tradierte, durch vielfältige Einflüsse weiterentwickelte System wird auch heutzutage in China "chinesische Medizin“ (Zhongyi) genannt, in Ergänzung zur westlichen Medizin (Xiyi; [1]). Der im Westen benutzte Begriff "Traditionelle Chinesische Medizin“ (TCM) wurde erst in den 1950er-Jahren geprägt. Er umfasst die Heiltraditionen Chinas, aber auch die Veränderungen und Weiterentwicklungen der TCM im 19. und 20. Jahrhundert [2-7].

Trotz der pluralistischen Wurzeln und Traditionen lassen sich wesentliche Grundpfeiler des Verständnisses von Gesundheit, Krankheit und Heilung als Kern der TCM definieren. Basis für die TCM ist ein eigenes diagnostisches System, das aufbauend auf Anamnese und qualitativen Befunden (Zunge, Puls, Palpitation) eine funktionelle Diagnose erhebt und eine differenzierte Terminologie für Physiologie und Pathologien (Normkonventionen, Agenzien, Leitkriterien, Funktionskreise usw.) nutzt [8, 9].

Konkrete Therapiemöglichkeiten sind die „fünf Säulen der TCM“:

1. Akupunktur,

2. chinesische Arzneimitteltherapie,

3. chinesische Diätetik und Lebenspflege,
In China wird TCM nach wie vor zur Behandlung von manchen akuten, häufig aber von chronischen und funktionellen Krankheiten verwendet. Wenig bekannt ist, dass Grundlagen der Hygiene, der erste Versuch einer Pockenimpfung und die Forensik in China als Teil der chinesischen Medizin historisch belegt sind [3-5]. Einige moderne Medikamente wie Ursodesoxycholsäure (UDCA), die Statine und Artemisinin (Standard gegen Malaria, Nobelpreis für Medizin 2015) stammen aus der chinesischen Medizin. Die TCM gilt heute als das umfassendste, am weitesten verbreitete traditionelle Medizinsystem.

\section{Geschichte und Rezeption im Westen}

Die westliche Welt kam im 17. Jahrhundert mit der chinesischen Medizin in Kontakt. Im Jahr 1683 verfasste der Niederländer Willem ten Rhijne, der als Arzt bei der Ostindischen Handelskompanie tätig war, einen ersten Bericht in westlicher Sprache über eine „NadelStich"-Therapie, die er in China kennengelernt hatte. Er führte den Begriff "Akupunktur" (,acus"= Nadel, „pungere" $=$ stechen) ein. Das bruchstückhafte Wissen, das anschließend in den Westen gelangte, führte dazu, dass man mit diesem Exotikum erste Versuche machte. Diese klinischen Versuche gingen sogar bis an die Pariser Sorbonne. Die Ergebnisse waren enttäuschend und so wurden diese Therapieversuche rasch aufgegeben.

War also chinesische Medizin nur ein Mythos und Chinoiserie?

Man hatte die Grundlagen, das Konzept der chinesischen Medizin, dessen Physiologie und Pathologie, in welches die Akupunktur eingebettet ist, nicht verstanden und übersetzt und somit auch keinen tieferen Zugang gewonnen. Die Inhalte, die Quellen und die Sprache der chinesischen Medizin mussten verständlich gemacht werden. So war es notwendig, zunächst die Aussagen der chinesischen Ärzte, das gesamte Paradigma der chinesischen Medizin sowie ihren philosophischen Hintergrund zu übermitteln.

Erst in den 1950er- bis 1970er-Jahren machten sich kenntnisreiche Sinologen daran, die große Sprachbarriere zu überwinden, wie Joseph Needham [4] oder Manfred Porkert mit seinem Standardwerk Die theoretischen Grundlagen der chinesischen Medizin [8] sowie Paul Unschuld [7]. Später erschienen auf Übersetzungen basierende, eher praktisch orientierte Werke wie zur systematischen Akupunktur (1984 [10]), zur chinesischen Arzneitherapie [11-14] sowie zur chinesischen Diätetik [15]. Diese ermöglichten ein erstes grundlegendes Verständnis der chinesischen Medizin.

Es bedurfte - wie in jeder Wissenschaft - einer verbindlichen Nomenklatur. In über 40-jähriger Arbeit entstand 2017 die "Standardnomenklatur der chinesischen Medizin“ [16], welche die Übertragung der TCM-Terminologie in mehreren westlichen Sprachen wiedergibt. Mit all diesem wurde also die sprachliche und verständnismäßige Grundlage geschaffen. 


\section{Entwicklung der TCM in China}

Von der Mitte des 19. bis zum Ende des 20. Jahrhunderts fanden in China extreme Umwälzungen politischer, wirtschaftlicher, kultureller und wissenschaftlicher Natur statt. Im Zuge dieser Entwicklung musste die chinesische Medizin zahlreiche Widerstände erdulden und ums Überleben kämpfen. Es war die Zeit, in der „die TCM in der Zwangsjacke absoluter Fehleinschätzung gefesselt war" (Li Zhichong, Direktor des chinesischen TCM-Verbandes, 2002 [2, 6]).

Nach der Gründung der Volksrepublik (VR) China 1949 wurde die sehr kritische Haltung der Kommunistischen Partei Chinas zur chinesischen Medizin aus politischen und wirtschaftlichen Gründen revidiert. Mao Zedongs berühmte Kalligrafie als Leitmotto lautete: "Chinesische Medizin ist ein großes Schatzhaus, das wir gründlich ans Licht holen und weiter entwickeln sollten" („Zhongguo yiyao xue shi yige weida baoku, yingdang nuli fajue jiayi tigao"). Dies wird zwar auch kritisch gewertet [7], führte aber zur Renaissance der TCM: In den 1950er-Jahren wurden zunächst in Beijing, Chengdu, Shanghai, Guangzhou und dann in allen anderen Provinzen TCM-Universitäten gegründet. Fünf berühmte Altärzte (Wulao) gaben inhaltlichen Input. 2000 westliche Ärzte mussten TCM studieren, das TCM-Konzept wurde systematisiert, standardisiert, kritisch der modernen Wissenschaft unterzogen und "gereinigt" $[1,6]$. Vor allem in den ersten Jahrzehnten der VR China war die TCM für die Gesundheitsversorgung unverzichtbar. Seit den 1990er-Jahren überwiegt aber die Rolle der westlichen Medizin. Im Jahr 1997 gab es ca. 250.000 TCM-Ärzte in China, aber über 1,2 Mio. westlich ausgebildete Ärzte.

Im letzten Jahrzehnt wurde von der chinesischen Regierung in hohem MaBe in Ausbildung und Forschung in der TCM mit den Zielen „Modernisierung“, „Normierung“ und „Integration der TCM“ investiert. Seit 2006 wurden TCM-Forscher in China, in Zusammenarbeit auch mit der Charité Berlin und der Technischen Universität München in modernen Forschungsstandards intensiv geschult. Die chinesische Regierung stellt derzeit umfassende Mittel zur Erforschung der TCM bereit. Die Forschung im Bereich Akupunktur, TCM und Komplementärmedizin hat seit 1998 weltweit auch mehr als 2 fach quantitativ im Vergleich zur biomedizinischen Forschung zugenommen, neben China kamen die meisten Studien aus dem Bereich aus Südkorea, Deutschland, Australien und Italien und wurden auch in Zeitschriften mit Peer-Review-Verfahren und gutem Impact Factor publiziert [54].

\section{Akupunktur als Teil der TCM}

Akupunktur wird seit 1996 von der Privaten Krankenversicherung in der Schmerztherapie erstattet. Seit 2004 ist sie bei chronischem Knieschmerz und Rückenschmerzen im Katalog der Gesetzlichen Krankenversicherung aufgenommen. Leitlinien $[17,18]$ empfehlen sie darüber hinaus bei Migräne, Rückenschmerzen und Kopfschmerzen. Die aktuelle Datenlage zur Akupunktur wird im Beitrag von Brinkhaus und Willich in diesem Themenheft ausführlich dargestellt.

Akupunktur und Moxibustion (Zhenjiu) sind Teil der TCM und werden für eine Vielzahl von Indikationen von akuten Infekten bis Zosterneuralgien im klinischen Alltag angewandt. Über Schmerz und Allergien hinaus gibt es eine große Zahl von Studien und Metaanalysen, die Datenlage ist aber uneinheitlich.

Die Arbeitsgruppe um J. MacDonald (Griffith University, Australien; [19]) wertete 159 Cochrane-Reviews und andere Metanalysen aus. Bei 8 Indikationen gab es eine klare positive Evidenz für Akupunktur (allergische Rhinitis, Migräneprophylaxe, Spannungskopfschmerz, Rückenschmerz, Knieschmerz, postoperativer Schmerz und Nausea sowie chemotherapieinduzierte Übelkeit). Bei 38 anderen Indikationen gab es positive randomisierte kontrollierte Studien (RCT, bspw. zur ambulanten Anästhesie, Menopause, Reizdarmsyndrom (IBS), nach Apoplexie), bei 71 war die Evidenz nicht ausreichend (bspw. Alzheimer, Tinnitus). Zudem gab es bei 5 Indikationen keinen Hinweis auf Wirksamkeit (bspw. Alkohol-, Nikotin- und Kokainentzug). Akupunktur war darüber hinaus kosteneffektiv und sicher [19].

Durch neue Verfahren (bspw. funktionelle MRT) konnten Hinweise auf neurologische Wirkmechanismen der Akupunktur bis hin zu einem „brain mapping" gewonnen werden: Eine Arbeitsgruppe um V. Napodov [20] stimulierte den Akupunkturpunkt Ma 36/St 36 und konnte im Vergleich zu Kontrollpunkten spezifische Aktivitäten im Parahippocampus und mittleren Gyrus nachweisen.

\section{(Traditionelle) chinesische Arzneimitteltherapie (TCA)}

Die traditionelle chinesische Arzneimitteltherapie (TCA) gilt in China als die wichtigste Therapiemethode der TCM. Als Voraussetzung für eine (richtige) Wirkung der TCA wird eine korrekte "chinesische Diagnose" nach den diagnostischen Verfahren der TCA $[9,11$, 12] erstellt, dann die Rezeptur ausgewählt, modifiziert und dem Patienten verordnet.

Grundprinzip ist die Kombination von 2-15 Arzneikräutern in einer individuellen Rezeptur. Die Rationalen sind Wirkverstärkung, Nutzung von Synergismen einzelner Arzneipflanzen, Lenkung der Rezepturmischung, Minderung von Geruchs- und Geschmacksbelästigung sowie unerwünschten Arzneiwirkungen. Klassisch werden die Mittel zusammen als Abkochung (Dekokt) verabreicht, moderne Applikationsformen sind Pulver, Granulierungen, Pillen und Sirupe. Vorausgehend werden die Arzneidrogen häufig vorbearbeitet (prozessiert, „paozhi“; [21]).

Voraussetzung sind die induktiv-empirische Systematik der (traditionellen) chinesischen Medizin [8,9] und des trotz aller Zeitläufe - bewahrten traditionellen Medizinwissens, weiter die Biodiversität Chinas (ca. $12 \%$ aller weltweit bekannten Pflanzenarten sind in China beheimatet) und der daraus resultierende hohe Anteil arzneilich nutzbarer Arten (ca. 700 offizielle Einträge in der Pharmakopöe der VR China 2005, Band 1 [22]). 
Bundesgesundheitsbl 2020 • 63:570-576 https://doi.org/10.1007/s00103-020-03132-9

(c) Der/die Autor(en) 2020

\section{C.-H. Hempen ·J. Hummelsberger}

\section{Traditionelle Chinesische Medizin (TCM) - vom Mythos zur Evidenz}

\section{Zusammenfassung}

Die Traditionelle Chinesische Medizin (TCM) ist heute das umfassendste, weltweit am weitesten verbreitete traditionelle Medizinsystem.

Ursprünglich erschien die TCM in unserem westlichen Kontext als ein seltsames, mythologisches Gebilde, jedoch wurde durch die Überwindung der sprachlichen Hürden ein Verständnis möglich. Physiologie, Diagnostik und die klinische Anwendung wurden anwendbar und validierbar. Im Jahr 1998 wurde durch das US-amerikanische National Insitute of Health erstmals eine Konsensuskonferenz zur Akupunktur durchgeführt. Seither werden Akupunktur und die TCM allgemein durch methodologisch gute Studien erforscht. Für die Akupunktur gibt es bereits Evidenz für die Wirksamkeit über Schmerztherapie und Allergie hinaus. Auch werden die Wirkmechanismen der Akupunktur bspw. durch Studien mit funktioneller Magnetresonanztomographie (fMRT) besser verstanden.

Auch für die chinesische Arzneitherapie (TCA) gibt es für einige Bereiche positive Metaanalysen und Studien. Hier besteht aber noch in großem Umfang Forschungsbedarf. Es zeigen sich Chancen für die Entwicklung neuer Medikamente und Rezepturen für die (Begleit-)Behandlung im Bereich von Infekten, Autoimmunkrankheiten, Krebs, Stoffwechsel-sowie dermatologischen und gastrointestinalen Krankheiten. Für die weitere Evaluierung der TCM, aber auch für eine integrative, kritische Anwendung in der Praxis der TCM ist eine fundierte
Ausbildung von Ärzten dringend erforderlich, eine weitere Institutionalisierung ist für die Patientensicherheit und Orientierung geboten.

Die TCM scheint aus der Grauzone des rein Mystischen herausgetreten zu sein, sie kann Chancen für eine bessere Patientenversorgung bieten. In vielen Teilen besteht und wächst die Evidenz für TCM, es ist aber weiter dringend umfassende Forschung erforderlich. Diese sollte unabhängig gefördert werden.

Schlüsselwörter

Chinesische Medizin · TCM · Akupunktur . Chinesische Arzneitherapie · Evidenz

\section{Traditional Chinese medicine (TCM)—what is myth and what is the state of evidence today?}

\section{Abstract}

Traditional Chinese medicine (TCM) is the most comprehensive and widely practiced system of medicine in the world.

Originally, TCM appeared in our Western context as an unfathomable mythological doctrine. Once the linguistic barriers had been overcome, it became possible to understand and apply the terminological and physiological concepts, the method of diagnosis, and the clinical findings.

Since the NIH consensus conference in 1998 our understanding of TCM has been the subject of methodologically robust studies. We now have evidence of the efficacy and effectiveness of acupuncture beyond its use in pain therapy and allergy treatment. Thanks to fMRI studies, among others, we also have a better understanding of the mechanisms underlying acupuncture.

Meta-analyses and studies also confirm the efficacy of Chinese medicinal drugs and remedies in some fields, yet there is still extensive need for further research. But opportunities are also emerging for new medicines and prescriptions for (accompanying) treatments of infections, autoimmune diseases, cancer, metabolic illnesses, dermatological disorders, and gastrointestinal diseases.

To enable further evaluation of TCM, but also to ensure an integrative, critical application in the practice of TCM, it is vital for doctors to have thorough training. Further institutionalisation is necessary to provide orientation and patient safety.

The days of regarding TCM as some kind of mysticism are indeed long over and it can offer real opportunities for better patient care. In many fields, there is increasing evidence of TCM's effectiveness, yet further comprehensive research is essential. This should be encouraged unconditionally.

\section{Keywords}

Chinese medicine - TCM - Acupuncture · Chinese herbal medicine $\cdot$ Evidence
Prinzipiell handelt es sich bei der TCA um eine allopathische Medizin. $\mathrm{Zu}$ den Inhaltsstoffen liegen umfangreiche Untersuchungen vor $[11,12]$. Wie in der Phytotherapie üblich, sind nur sehr selten einzelne Wirkstoffe identifizierbar (beispielsweise UDCA, Artemisinin, Berberin, Baikalin). Vielmehr sind Wirkstoffgemische für die Wirkung verantwortlich. Diese Wirkstoffgemische entfalten nur als solches den Effekt (analog z. B. zum Johanniskrautextrakt). Zur Materia Medica, zu den Rezepturen und zum System der TCA sind Standardwerke erhält- lich. Derzeit erarbeitet die Europäische Arzneimittelagentur (EMA) europäische Monografien chinesischer Arzneidrogen. In einem interdisziplinären Projekt unter Führung der Landesanstalt für Landwirtschaft Weihenstephan wird der Anbau von TCA-Pflanzen erforscht. Aktuell können mehr als 20 Arzneipflanzen unter kontrollierten Bedingungen auch hier kultiviert werden. Dabei wurden umfassende Kenntnisse zu Inhaltsstoffen, Identität und Sicherheit gewonnen [23].

\section{Wirkweise und Studienlage zur Wirksamkeit der TCA}

$\mathrm{Zu}$ Wirkungen und Wirksamkeit der TCA ist inzwischen eine Vielzahl von Studien und Reviews erschienen. Aufgrund der großen Breite der Rezepturen/ Wirkstoffe und Indikationsvielfalt sind diese aber sehr widersprüchlich.

Die traditionellen Wirkprinzipien der TCA (Verordnung nach TCM-Diagnosen) sind nur in wenigen Studien, die den aktuellen Standards entsprechen, überprüft worden: In dem 1998 im Jour- 


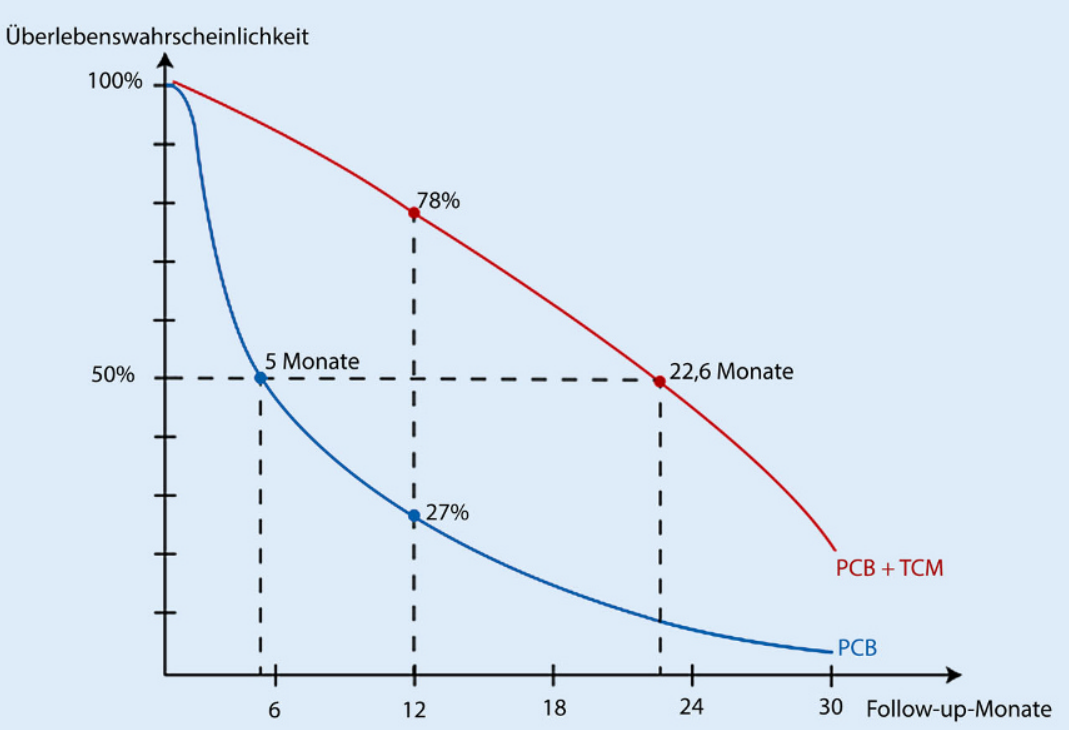

Abb. 1 A Überlebenswahrscheinlichkeit bei fortgeschrittenem Adenolungenkarzinom Stadium 4 bei platinbasierter Chemotherapie (PCB) und bei zusätzlicher TCM-Behandlung. Untersucht wurden insgesamt 133 Patienten [29]

nal of the American Medical Association (JAMA) veröffentlichen RCT zum Reizdarm [24] wurden doppelblind sowohl eine TCA-Standardrezeptur als auch nach TCA-Kriterien individualisierte Rezepturen mit Placebo verglichen. Nach 16 Wochen waren beide Verumgruppen signifikant zum Placebo gebessert. Im Follow-up hielt der Besserungseffekt bei der Individualgruppe an, bei der Standardrezepturgruppe kam es häufiger zum Rückfall.

\section{Wirkprinzipien nach westlichen pharmakologischen Modellen}

$\mathrm{Zu}$ den Wirkprinzipien nach westlichen pharmakologischen Modellen liegen zahlreiche Untersuchungen vor, sowohl in vitro als auch an Tiermodellen. Insgesamt konnten allein durch eine Literaturrecherche in Pubmed aktuell 16.356 (Abfrage am 01.10.2019) Einträge dazu gefunden werden.

Asthma. In einer Studie zur Behandlung von Asthma mit TCA wurden immunmodulatorische und antiinflammatorische Effekte sowie eine relaxierende Wirkung auf die Atemwegsmuskulatur einer Rezeptur aus Ganoderma, Sophora- und Astragalus-Wurzeln („ASHMI“) nachgewiesen. Der Cough Symptom Score CSS $4,7(3,5-5,7)$ vorher, wurde eine
Verbesserung auf dann 0,9 (0,14-2,3) nachgewiesen, die nach 4 Wochen einer Verbesserung mit einer Prednisontherapie (CSS vorher 4,7, nachher 0,6) gleichwertig waren. Allerdings nahmen die Patienten in der Prednisongruppe $2,4 \mathrm{~kg}$ zu. Nach 3 Monaten Einnahme der ASHMI-Medikation nahm das Serum-IgE ab und der Serum-InterferonGamma-Spiegel zu [25].

Krebserkrankungen. Aktuelle Studien und Reviews zur TCA weisen auf eine unterstützende Wirkung bei Krebserkrankungen hin. Eine Metanalyse zu Astragali Radix (Huangqi) zeigt eine unterstützende Wirkung bei Chemotherapien beim kleinzelligen Bronchialkarzinom. In 12 Studien wurde ein reduziertes Sterberisiko in 12 Monaten gefunden (Risk Ratio $\mathrm{RR}=0,67$, KI $95 \%$ : 0,52-0,87), in 30 Studien eine verbesserte Tumorresponse (RR 1,34, KI 95\%: 1,24-1,46; [26]). Es traten keine vermehrten Nebenwirkungen oder Interaktionen mit der platinbasierten Chemotherapie auf. Postulierte Mechanismen sind Stimulierung der Makropagen- und T-Killerzellaktivität sowie Interleukin(IL)-Inhibition. Berberin, ein Hauptbestandteil der Arzneidrogen Coptis Rhizoma (Huanglian) und Scutellariae Radix (Huangqin), ist ein Breitspektrumenzyminhibitor und weist antiproliferative wie proapoptotische Effekte auf [27].

Atopische Dermatitis. In mehreren, wenn auch kleinen RCTs wurde eine Wirksamkeit der TCA bei atopischer Dermatitis gesehen. In einer weiteren Studie wurden als immunologisches Korrelat reduzierte Serum-IgE-Komplexe, IL-4-Aktivität und IL-2-Rezeptorzellaffinität gefunden [28].

\section{Colitis/chronisch entzündliche Darmer-} krankung (CED). Bei Colitis/CED scheinen die benutzten Arzneidrogen antiinflammatorische Effekte zu haben.

Zusammenfassend gibt es bereits zahlreiche Publikationen zu den Wirkmechanismen und -prinzipien. Insbesondere wurden bisher immunmodulatorische, antiinflammatorische, antitumoröse Eigenschaften aufgezeigt. Dementsprechend kann angenommen werden, dass auch bei allergischen und immunologischen Erkrankungen (Asthma, Neurodermitis), komplementär zur Tumortherapie, bei entzündlichen Darmerkrankungen sowie funktionellen Störungen pharmakologische Prinzipien anwendbar sind.

Aufgrund der Vielzahl der Einzelarzneien und Rezepturen, der Inhaltsstoffe, der Wirkstoffgemische und möglicher Indikationen ist jedoch weitere umfassende Forschung zwingend erforderlich.

\section{Daten zur Wirksamkeit von TCA}

Randomisierte kontrollierte Studien (RCT) gibt es u.a. zum IBS, (komplementär) beim Pankreaskarzinom, zu Ginseng bei karzinomassoziierter Fatigue und, wie in $\mathbf{A b b} 1$ dargestellt, bei fortgeschrittenem Bronchialkarzinom mit platinbasierter Chemotherapie [29]. Hier zeigte sich, dass die Lebensqualität (EOCG- und FACT-1-Scores) dieser Patientengruppe durch zusätzliche Gabe von TCA im Vergleich zu einer Placebokräutergruppe deutlich verbessert werden konnte.

Für die TCA liegen Cochrane-Reviews bzw. Metaanalysen mit positiven Hinweisen auf die Wirksamkeit bspw. zu folgenden Indikationen und Diagnosen vor: 
- In-vitro-Fertilisation (IVF): Die Kombination aus TCA und IVF erhöhte signifikant die Fertilitätsraten (OR 2,04, $95 \%$ KI: 16,67-2,40, $p<0,0001 ;[30])$.

- Nephrotisches Syndrom: Astragali Radix führte zu einer Verbesserung der Albuminausscheidung (0,57 g/24h, $95 \% \mathrm{KI}:-1,04--0,10)$ und des Allgemeinprogresses beim nephrotischen Syndrom [31].

- Schizophrenie: Für die Rezeptur „Dekokt, das den Fk Gallenblase erwärmt" (Wendan Tang) bei Schizophrenie, die komplementär zu Antipsychotika gegeben wurde, liegen Wirksamkeitsnachweise vor [32, 33].

- Akute Infekte der Atemwege [34]: Hier war das Forschungsteam durch die verschiedenen TCA-Diagnosen (siehe Differenzialtherapie abhängig vom Syndrommuster) und dadurch verschiedene Rezepturen aber nicht in der Lage, eine einheitliche Rezeptur zu filtern.

- Postoperative Behandlung von Endometriose: TCA führte zu einer deutlichen Verbesserung und Rückgang von Adnexherden als Danazol (RR 1,70, $95 \%$ KI: 1,04-2,78; [35]).

- Primäre Dysmenorrhö: TCA führte zur einer Schmerzlinderung (14 RCTs; RR 1,99, 95 \% KI: 1,52-2,60), Verbesserung des Allgemeinzustandes (6 RCTs; RR 2,17, $95 \% \mathrm{KI}: 1,73-2,73)$ verglichen $\mathrm{zu}$ anderen Schmerzmitteln [36].

- Pathologische Glukosetoleranz: Durch TCA in Kombination mit Lebensstiländerungen normalisierten sich die Blutzuckerwerte doppelt so häufig, als wenn lediglich Lebensstiländerungen durchgeführt wurden (RR 2,07; $95 \%$ KI: 1,52-2,82). Die TCA-Gruppe hatte einen deutlich selteneren Progress im Vergleich zu Vollbilddiabetes (RR 0,33; $95 \% \mathrm{KI}$ : 0,19-0,58; [37]).

- Atopische Dermatitis: Es gibt Hinweise zur Wirksamkeit in $[28,38]$.

- Prämenstruelles Syndrom: Nachweise von Wirksamkeit bei der Symptomreduktion (RR 3,50, 95 \% KI: 1,74-7,06) gibt es in [39].
- Nebenwirkungen durch Chemotherapie bei Karzinom: Zur Reduktion von Übelkeit (Nausea) und anderen Nebenwirkungen wie Leukopenie gibt es Hinweise zur Wirksamkeit von Astragali Radix in [40].

- Palliativversorgung: Zur Unterstützung in der Palliativversorgung zur Schmerzreduktion (Schmerzreduktion 3 RCTs, $95 \% \mathrm{KI}:-1,69$ bis $-0,11$ ), Verstopfung und Fatigue [41, 42].

- Nichtkleinzelliges Bronchialkarzinom (komplementär): [26, 42].

- Akute Pankreatitis (komplementär): [43].

- Akute und chronische Harnwegsinfekte: Es kam zu weniger Rückfällen bei akuten (RR 1,21, 95 \% KI: 1,11-33) und bei chronischen Harnwegsinfekten (RR 0,53, KI $95 \%$ : 0,35-0,80; [44]).

\section{Kritik und negative Studienlage zu TCA}

Negative Metaanalysen (Cochrane-Reviews u.a.) liegen u.a. zu HIV/Aids, Diabetes mellitus Typ 2, Hepatitis C (mit Einschränkungen) sowie Uterusfibromen vor $[45,46]$.

Generell werden bei den aus China stammenden Studien die Größe, Berichtsqualität, Heterogenität, mangelnde Vergleichbarkeit sowie unklare Angaben insbesondere zur Randomisierung kritisiert und dadurch eine mögliche Verzerrung (Bias) kritisch bewertet [47].

Methodenkritisch muss bei Bewertung der TCA ausschließlich durch RCTs die asymmetrische Aussagekraft der RCTs in Betracht gezogen werden. Während ein positives Ergebnis Beweiskraft besitzt, ist ein negatives Ergebnis einer RCT kein negativer Beweis. („The major weakness of the randomized controlled trial is the difficulty for protection against false negativity“ $[48,49]$.) Das Design der RCT ist für manche Fragestellungen ungeeignet. Fallkontrollstudien, prospektive Kohortenstudien, Serien von Einzelfallberichten, Konsensuskonferenzen von Experten u. a. scheinen speziell auch im Fall der TCA mit der sehr komplexen Differenzialdiagnose und den Therapiestrategien besser geeignet, um weitere Evidenz (positiv wie negativ) zu gewinnen.
Sicherheits- und Qualitätsaspekte der TCA

Trotz negativer Medienberichte ist festzustellen, dass Nebenwirkungen bei korrekter Anwendung, Sicherung der Arzneiqualität (Prüfung auf Verunreinigungen und Identität, Abgabe durch Apotheken) nach aktuellem Wissensstand selten sind. Das Zentrum für Therapiesicherheit in der TCA (www.ctca.de) konnte in den letzten 15 Jahren nachweisen, dass nur in wenigen Fällen Anstiege der Leberwerte auftraten, die aber reversibel waren. Weitere Nebenwirkungen waren Durchfall, Allergien, idiosynkratische Reaktionen und Interaktionen mit westlichen Medikamenten. Sicherheitsrisiken und Qualitätsmängel entstehen in der TCA, wenn - chinesische Arzneimittel über das Internet oder den Graumarkt erworben werden,

- vorgegebene Standards zur Identitätssicherung, Qualität und Reinheit der Arzneidrogen nicht eingehalten werden sollten, was aber bei Abgabe durch Apotheken ausgeschlossen sein sollte,

- die verschreibenden Ärzte nicht ausreichend ausgebildet sind.

Einen Überblick über den Stand der Therapiesicherheit geben Publikationen von Hempen [50] und Wiebrecht [51].

\section{Taijiquan und Qigong - Aktuelle Studien und Metaanalysen}

Taiji und Qigong sind Bewegungstherapien, die in chinesischen Kampfkünsten und in den Lehren zur Lebenspflege (Yangsheng) wurzeln. Geregelte Bewegungsabfolgen werden mit imaginativen Techniken, Atemübungen und meditativen Elementen verknüpft. Seit den 1950er-Jahren werden diese in China in speziellen Kliniken wie im Alltag angewandt. Auch im Westen sind sie weitverbreitet und werden gelehrt.

Mittlerweile gibt es ca. 500 Studien und eine Vielzahl von Metaanalysen, die zum Teil hochrangig publiziert wurden. Die besten Belege für positive Wirkungen gibt es in der Begleitbehandlung bei Morbus Parkinson [52], da sie Gehfähigkeit, Koordination und motorische Fähigkei- 
ten verbessern und möglicherweise das Voranschreiten der Erkrankung verlangsamen. Positive Effekte konnten auch bei Arthrose, Osteoporose, Prophylaxe von Stürzen bei Älteren, chronisch obstruktiver Lungenkrankheit (COPD) und hinsichtlich des Atemvolumens nachgewiesen werden.

Die Anwendung von Taijiquan bei Demenz wurde mit der Methode „systematic review and meta-analysis" [53] untersucht. Es erfolgte eine Auswertung von insgesamt 20 Studien verschiedensten Designs mit insg. 2553 Teilnehmern. Hier konnte bei Taijiquan-Anwendern eine signifikante, wenn auch nur geringe Verbesserung der kognitiven Funktionen (im MMSE-Test, Mini-Mental-State Examination) im Vergleich zu den Kontrollgruppen mit anderen aktiven Interventionen (Effektgrößen $=$ Hedges $g=0,30$, $=0,002, \mathrm{I} 2=0 \%)$ und ohne aktive Interventionen nachgewiesen werden. In einer der Studien konnte eine deutlich reduzierte Progression einer Demenz bei der Taiji-Gruppe mit 4,3\% Progress gegenüber der Gruppe mit „westlichen Übungen“" mit 16,6\% Progress im Beobachtungszeitraum dokumentiert werden.

\section{Chinesische Diätetik, Tuina}

Diätetik und Lebenspflege dienen vergleichbar mit den Ordnungstherapien in der westlichen Naturheilkunde zur Verbesserung der Lebensführung. Tuina wirkt analog zur westlichen manuellen Medizin. $\mathrm{Zu}$ diesen Bereichen gibt es eine Vielzahl von Anwendungsbeobachtungen, allerdings liegen keine RCTs oder anderen aussagefähigen Studien vor.

\section{Weitere Perspektiven und ärztliche Ausbildung}

Voraussetzung für eine wirksame Anwendung muss eine umfassende Ausbildung auf der Basis ärztlichen Wissens sein.

Ärzte, die TCM und Akupunktur anwenden, müssen auch im sprachlichen und sozialen Kontext die Methoden der TCM in den Rahmen unserer westlichen Medizin integrieren können, auch um die
Möglichkeiten und Grenzen für das Wohl der Patienten auszuschöpfen.

In den vergangenen Jahrzehnten wurden durch ärztliche Fachgesellschaften umfassende Ausbildungsmöglichkeiten geschaffen. Nach Angaben der Bundesärztekammer (BÄK) hatten zum 31.12.2018 insgesamt 14.648 Ärzte die Zusatzbezeichnung Akupunktur erworben (https://www.bundesaerztekammer. de/ueber-uns/aerztestatistik/aerztestatis tik-2018/). Es ist davon auszugehen, dass die meisten die Methode regelmäßig anwenden. Circa 2000 Ärzte verschreiben chinesische Arzneimittel. Leider gibt es keine validen Angaben zum Bereich der Heilpraktiker.

Akademisch wird die Ausbildung durch die Einrichtung des Masterstudiengangs TCM (M. Sc.) an der Technischen Universität München seit 2011 als berufsbegleitender, 6-semestriger Masterstudiengang (Regelstudienzeit $3600 \mathrm{~h}$ ) nach den Bologna-Kriterien angeboten. Ein analoger Studiengang wurde 2018 an der Universität Hamburg-Eppendorf zusammen mit der Universität Shanghai installiert, weitere Studiengänge sind im europäischen Ausland in Planung, als Wahlpflichtfach wird TCM bspw. in Witten-Herdecke, München und Erlangen angeboten.

\section{Implementierung von TCM- Diagnosen im ICD-11}

Die neue Internationale Klassifikation der Krankheiten (ICD-11) soll am 01.01.2022 in Kraft treten. Diagnosen und Syndrome gemäß der Traditionellen Chinesischen Medizin finden sich dort in Kap. 26. Es werden 470 chinesische Diagnosen, Syndrome und Zustände genannt. Hiermit wird fast die gesamte TCM im ICD-11 wiedergegeben und die ärztliche Codierung von TCM-Diagnosen wird so möglich. Dies ist einer zukünftigen Erforschung der TCM dienlich. Da die TCM-Diagnosen aber keine westlichen Diagnosen ersetzten können und mit diesen nicht vergleichbar sind, muss diese Entwicklung auch kritisch bewertet werden.

\section{Korrespondenzadresse}

Prof. Dr. med. Carl-Hermann Hempen

Technische Universität München

Georg-Brauchle-Ring 62, 80992 München, Deutschland

ch@hempen.de

Funding. Open Access funding provided by Projekt DEAL.

\section{Einhaltung ethischer Richtlinien}

Interessenkonflikt. J. Hummelsberger erhält Honorare als Dozent für Akupunktur und TCM an der TU München sowie von verschiedenen ärztlichen Fachgesellschaften. Er war bis März 2020 im Vorstand der Internationalen Gesellschaft für Chinesische Medizin (SMS, Societas Medicinae Sinensis), München. Verbindungen zu Unternehmen bestehen nicht. C.-H. Hempen gibt an, dass kein Interessenkonflikt besteht.

Für diesen Beitrag wurden von den Autoren keine Studien an Menschen oder Tieren durchgeführt. Für die aufgeführten Studien gelten die jeweils dort angegebenen ethischen Richtlinien.

Open Access. Dieser Artikel wird unter der Creative Commons Namensnennung 4.0 International Lizenz veröffentlicht, welche die Nutzung, Vervielfältigung, Bearbeitung, Verbreitung und Wiedergabe in jeglichem Medium und Format erlaubt, sofern Sie den/die ursprünglichen Autor(en) und die Quelle ordnungsgemäß nennen, einen Link zur Creative Commons Lizenz beifügen und angeben, ob Änderungen vorgenommen wurden.

Die in diesem Artikel enthaltenen Bilder und sonstiges Drittmaterial unterliegen ebenfalls der genannten Creative Commons Lizenz, sofern sich aus der Abbildungslegende nichts anderes ergibt. Sofern das betreffende Material nicht unter der genannten Creative Commons Lizenz steht und die betreffende Handlung nicht nach gesetzlichen Vorschriften erlaubt ist, ist für die oben aufgeführten Weiterverwendungen des Materials die Einwilligung des jeweiligen Rechteinhabers einzuholen.

Weitere Details zur Lizenz entnehmen Sie bitte der Lizenzinformation auf http://creativecommons.org/ licenses/by/4.0/deed.de.

\section{Literatur}

1. Despeux C, Wislsperger G (2000) Zur Geschichte der chinesischen Medizin. Von der Frühzeit in die Moderne. U\&V, München

2. Frühauf H, Leeb S (2005) Chinesische Medizin in der Krise: Wissenschaftliche und politische Hintergründe der Entstehung der "TCM“ (Teil 1: 1850-1990). U\&V, München, S1-12

3. Ho P-Y, Lisowski F-P (1997) A brief history of Chinese Medicine. World Scientific, Singapore, New York, London

4. Lu G-D, Needham J (2002) Celestial lancets. A history and rationale of acupuncture and moxa. Routldege, Oxon 
5. Needham J, Lu G-D, Sivin N (2004) Science and Civilisation in China. Cambridge University Press, Cambridge (Vol 6: Biology and Biological Technology, Part VI: Medicine)

6. Scheid V (2007) Currents of tradition in Chinese Medicine, 1626-2006. Eastland Press, Seattle

7. Unschuld P-U (1985) Medicine in China-a history of ideas. University of California Press, Berkeley, Los Angeles, London

8. Porkert M (1991) Die theoretischen Grundlagen der chinesischen Medizin. AMS, Basel

9. Porkert M (1993) Neues Lehrbuch der chinesischen Diagnostik. Phainon, Dinkelscherben

10. Porkert M, Hempen C-H (1985) Systematische Akupunktur. Urban \& Schwarzenberg, München, Wien, Baltimore

11. Hempen $\mathrm{C}-\mathrm{H}$, Fischer $\mathrm{S}$, Hummelsberger J (2006) Leitfaden Chinesische Rezepturen. Elsevier, München

12. Hempen $\mathrm{C}-\mathrm{H}$, Fischer T (2006) Leitfaden ChinesischePhytotherapie. Elsevier, München

13. Bensky D, Barolet A (2005) Chinese herbal medicine-Materia medica. Eastland Press, Seattle

14. Scheid V, Bensky D, Stöger E (2009) Chinese herbal medicine. Formulas and strategies. Eastland Press, Seattle

15. Engelhardt U, Hempen C-H (1997) Chinesische Diätetik. Urban \& Schwarzenberg, München

16. World Federation of Chinese Medicine Societies (2017) Übersetzung der internationalen Standardnomenklatur der chinesischen Medizin in Chinesisch-Deutsch. People's Medical Publishing House, Peking

17. AWMF (2019) Leitlinien Therapie der Migräneattacke und Prophylaxe. https://www.awmf.org/ uploads/tx_szleitlinien/030-057l_S1_MigraeneTherapie_2019-10.pdf.Zugegriffen: 13.Jan. 2020

18. AWMF (2018) Leitlinie Rückenschmerz. https:// www.awmf.org/uploads/tx_szleitlinien/nvl007k_S3_Kreuzschmerz_2018-02.pdf\#page =1\& zoom=auto,-14,843.Zugegriffen: 13.Jan. 2020

19. MacDonald J (2017) The acupuncture evidence project. https://www.acupuncture.org. au/wp-content/uploads/2017/11/28-NOV-TheAcupuncture-Evidence-Project_Mcdonald-andJanz_-REISSUED_28_Nov.pdf. Zugegriffen: 29. Dez. 2019

20. Long X, Huang W, Napodov V et al (2016) Sustained effects of acupuncture stimulation investigated with centrality mapping analysis. Front Hum Neurosci 10:510

21. Hu C-J, Nögel R, Hummelsberger J, Engelhardt U (2019) Paozhi - Die Aufbereitung chinesischer Arzneimittel. Springer, München

22. Guojia yaodian weiyuanhui (2005) Pharmakopöe der VR China. Chinesischer Verlag für Medizin, Pharmakologie, Wissenschaft und Technik, Peking

23. Hummelsberger J, Bomme U, Friedl F (2006) Chinesische Heilpflanzen - Anbau hierzulande garantiert Qualität. Dtsch Ärztebl 103:21

24. Bensoussan A, Talley NJ, Hing M, Menzies R, Guo A, Ngu M (1998) Treatment of irritable bowel syndrome with Chinese herbal medicine: a randomized controlled trial. JAMA 280:1585-1589

25. Li XM, Brown L (2009) Efficacy and mechanisms of action of traditional Chinese medicines for treating asthma and allergy. J Allergy Clin Immunol 123:297-308

26. McCulloch M, See C, Shu XJ et al (2006) Astragalus-based Chinese herbs and platinumbased chemotherapy for advanced non-small-cell lung cancer: meta-analysis of randomized trials. JClin Oncol 24:419-430
27. Sun Y, Xun K, Wang Y, Chen X (2009) A systematic review of the anticancer properties of berberine, a natural product from Chinese herbs. Anticancer Drugs 20:757-769

28. Sheehan MP, Rustin MH, Atherton DJ et al (1992) Efficacy of traditional Chinese herbal therapy in adult atopic dermatitis. Lancet 340:13-17

29. Guo H, Liu J-X, Li H, Baak J-PA (2017) In metastatic non-small cell lung cancer platinum-based treated patients, herbal treatment improves the quality of life. A prospective randomized controlled clinical trial. Front Pharmacol 8:454

30. Cao HJ, Han M, Ng EH et al (2013) Can Chinese herbal medicine improve outcomes of IVF? A systematic review and metaanalysis of RCT's. PLoS One 8:12

31. Yuan W, Wang J, Wu T (2008) Chinese herbal medicine Huangqi type formulations for nephrotic syndrome. Cochrane Database Syst Rev 2:CD6335

32. Rathbone J, Zhang L, Zhang M et al (2005) Chinese herbal medicine for schizophrenia. Cochrane Database Syst Rev 4:CD3444

33. Deng H, Xu J (2017) Wendan decoction for schizophrenia. Cochrane Database Syst Rev 6:CD12217

34. Zhang X, Wu T, Zhang J, Yan Q, Xie L, Liu G-J (2007) Chinese medicinal herbs for the common cold. Cochrane Database Syst Rev 1:CD4782

35. Flower A, Liu JP, Chen S, Lewith G (2009) Chinese herbal medicine for endometriosis. Cochrane Database Syst Rev 3:CD6568

36. Zhu X, Proctor $M$, Bensoussan A, Smith CA Wu E (2007) Chinese herbal medicine for primary dysmenorrhoea. Cochrane Database Syst Rev 4:CD5288

37. Grant S,Bensoussan A, Chang Detal (2009)Chinese herbal medicines for people with impaired glucose tolerance or impaired fasting blood glucose. Cochrane Database Syst Rev 4:CD6690

38. Zhang W, Leonard T, Bath-Hextall F, Chambers CA Lee C, Humphreys R, Williams HC (2004) Chinese herbal medicine for atopic eczema. Cochrane Database Syst Rev 4:CD2291

39. Jing Z, Yang X, Ismail K, Chen X et al (2009) Chinese herbal medicine for premenstrual syndrome. Cochrane Database Syst Rev 1:CD6414

40. Adams D, Wu T, Yang X et al (2009) Traditional Chinese medicinal herbs for the treatment of idiopathic chronic fatigue and chronic fatigue syndrome. Cochrane Database Syst Rev 4:CD6348

41. Barton DL, Soori GS, Bauer BA et al (2010) Pilot study of Panax quinquefolius (American ginseng) to improve cancer-related fatigue: a randomized, double-blind, dose-finding evaluation: NCCTG trial N03CA. Support Care Cancer 18:179-187

42. Chung V-CH, Wu X-Y, Lu P et al (2016) CHM for symptome management in cancer palliative care. Medicine 95:7

43. Wang G, Guo Z, Zhao P, Wang Y, Gan T, Yang J (2005) Chinese herbal medicines for acute pancreatitis. Cochrane Database Syst Rev 1:CD3631

44. Flower A, Wang L-Q, Lewith G, Liu J-P, Li Q (2015) Chinese herbal medicines for treating recurrent urinary tract infections in women. Cochrane Database Syst Rev 6:CD10446

45. Liu JP, Zhang M, Wang W, Grimsgaard S (2002) Chinese herbal medicines for type 2 diabetes mellitus. Cochrane Database Syst Rev 3:CD3642

46. Liu JP, Manheimer E, Yang M (2005) Herbal medicines for treating HIV infection and AIDS. Cochrane Database Syst Rev 3:CD3937

47. Manheimer E, Wieland S, Kimbrough E, Cheng K, Berman B-M (2009) Evidence from the Cochrane
Collaboration for Traditional Chinese Medicine therapies. J Altern Complement Med 9:1001-1014 48. Altman G-D, Bland J-M (1995) Absence of evidence is not evidence of absence. BMJ 311:485

49. Freireich E-J (1997) The randomized controlled trial is not the best and certainly not the only way to conduct clinical research. Adv J Mind Body Health 13:41-44

50. Hempen A, Huber R (2014) Qualität und Sicherheit chinesischer Arzneidrogen in Deutschland - ein Update. Forsch Komplementmed 21:401-412

51. Wiebrecht A (2017) Sicherheit in der Chinesischen Arzneitherapie. In: Focks C (Hrsg) Leitfaden Chinesische Medizin - Grundlagen. Elsevier, München

52. Ni X, Liu S, Lu F, Shi X, Guo X (2014) Efficacy and safety of Tai Chi for Parkinson's disease: a systematic review and meta-analysis of randomized controlled trials. PLoSOne 9:6

53. Wayne P-M, Walsh J-N, Taylor-Piliae R-E (2014) Effect of Tai Chi on cognitive performance in older adults: systematic review and meta-analysis. J Am Geriatr Soc 62:25-39

54. o A (1998) NIH Consensus Conference. Acupuncture. JAMA 280(17):1518-1524 